\title{
Checking and Interpolation of Functions Tabulated at Certain Irregular Logarithmic Intervals
}

\author{
Herbert E. Salzer
}

\begin{abstract}
Many functions $f(x)$ behave as polynomials in $\log x$. When tabulated for arguments in geometric progression, $f(x)$ can be checked by ordinary differencing, and interpolation can be performed to a fine extent with existing tables of Lagrangian coefficients. But in practice, $f(x)$ is often known or calculated at some or all of the points $1,2,5,10,20,50,100,200,500$, and 1000 (same theory for the points $0.001,0.002,0.005,0.01$, etc., or $0.01,0.02,0.05,0.1$, etc., or any constant multiple of $1,2,5, \ldots$.).

The present tables have a twofold use: I. Checking the correctness of $f(x)$ when tabulated at some of the more frequently occurring combinations of points $1,2,5$, etc. This also includes their use to estimate the least number of tabular entries for interpolation of given accuracy. II. Facilitation of Lagrangian interpolation by a generalization of a scheme given by $W$. J. Taylor.
\end{abstract}

\section{Introduction}

Functions which behave like polynomials in $\log x$ are encountered in numerous fields, such as statistics, actuarial studies, economics, biometrics, electronics, nuclear physics, biophysics, physical chemistry, etc. When a function obtained experimentally is suspected to have this form, it is usual to examine this by plotting the values on semilogarithmic graph paper and observing whether they lie on a smooth curve. When it has been decided that the function is approximately a polynomial in $\log x$ (or when it is known to be exactly a polynomial in $\log x$ ), the question arises as to how this information can be used to facilitate checking the table and interpolating in it numerically. This is the question to be discussed here.

\section{Arguments in Geometric Progression}

If the tabular arguments are in geometric progression, such as $1,2,4,8, \ldots$; or $1,10,100,1000$, the problems of checking and interpolation are quite simple, because the function is effectively tabulated at a constant interval in $\log x$. Thus an examination of the ordinary differences of the tabular values will reveal any errors, as well as the lowest degree which an approximating polynomial in $\log x$ must have in order to yield a certain desired accuracy. Either the differences themselves can be used for interpolation, or if we wish to avoid using differences, any one of several well-known tables of Lagrangian interpolation coefficients may be used for interpolation to a very fine subdivision of the tabular interval. The most extensive of these tables of interpolation coefficients are contained in [1]. ${ }^{1}$

\section{Divided Differences for Checking}

In practice, however, it is often found that the function has been determined at some or all of the points $1,2,5,10,20,50,100, \ldots, 1000$ (or a constant multiple of those values, e. g., $0.1,0.2,0.5$, $1.0, \ldots$. . . Then it is no longer possible to use the

${ }^{1}$ Figures in brackets indicate the literature references at the end of this paper. ordinary differences for checking purposes. Instead we use a certain generalization, known as "divided differences," a subject that is treated fully in most textbooks on finite differences (see [2] or [3]). The $(n-1)$ th divided difference with respect to $\log x$, of a function $f(x)$ tabulated at the $\mathrm{n}$ points $x_{1}, x_{2}, \ldots$, $x_{n}$, can be written in the form $\sum_{i=1}^{n} A_{i} f\left(x_{i}\right)$, where the $A_{i}$ are certain numbers depending only on the $x_{i}$, not on the function. It is convenient to tabulate these quantities, once and for all, for the usual sets of values of $x_{i}$ and for the various values of $n$, so that the divided difference can be obtained by a single accumulation on a calculating machine. This has been done here for $n=3(1) 10$ and $x_{i}$ ranging from $1,2,5, \ldots$, to 1000 , in table $1, \mathrm{~A}$; for $n=3(1) 7$ and $x_{i}$ ranging from $1,5,10, \ldots$ to 1000 in table $1, \mathrm{~B}$; and for $n=3(1) 7$ and $x_{i}$ ranging from $1,2,10$, . . . to 1000 in table 1, C. All entries are given to eight significant figures, correct to about a unit in the last place.

The explicit expression for $A_{i}$ is

$$
A_{i}=1 / \pi_{j}^{\prime}\left(\log _{10} x_{i}-\log _{10} x_{j}\right),
$$

where $\pi_{j}^{\prime}$ indicates the product over all $j \neq i$. It can be shown that if $f(x)$ is a polynomial of degree $n-2$ in $\log x$, then the $(n-1)$ th divided difference is identically zero. If the $(n-1)$ th divided difference is sufficiently small, then $f(x)$ considered as a polynomial of degree $n-2$ in $\log x$, is in all likelihood, free from error. (However the user is cautioned that a sufficiently small value of $\sum_{i=1}^{n} A_{i} f\left(x_{i}\right)$ is only a strong indication, rather than conclusive evidence, of the correctness of the $n$ entries, considered as values of a polynomial of degree $n-2$.) Now the tabular entries may be entirely correct and the user might still want to employ the most economical Lagrangian formula. Again, this information is conveyed in the sufficiently small $(n-1)$ th divided difference, which establishes the adequacy of an $(n-1)$ point Lagrangian interpolation formula. In brief, if the function is checked 
TABLE 1. Coefficients $A_{i}$ for checking and interpolation, based upon the points $x_{i}$ A

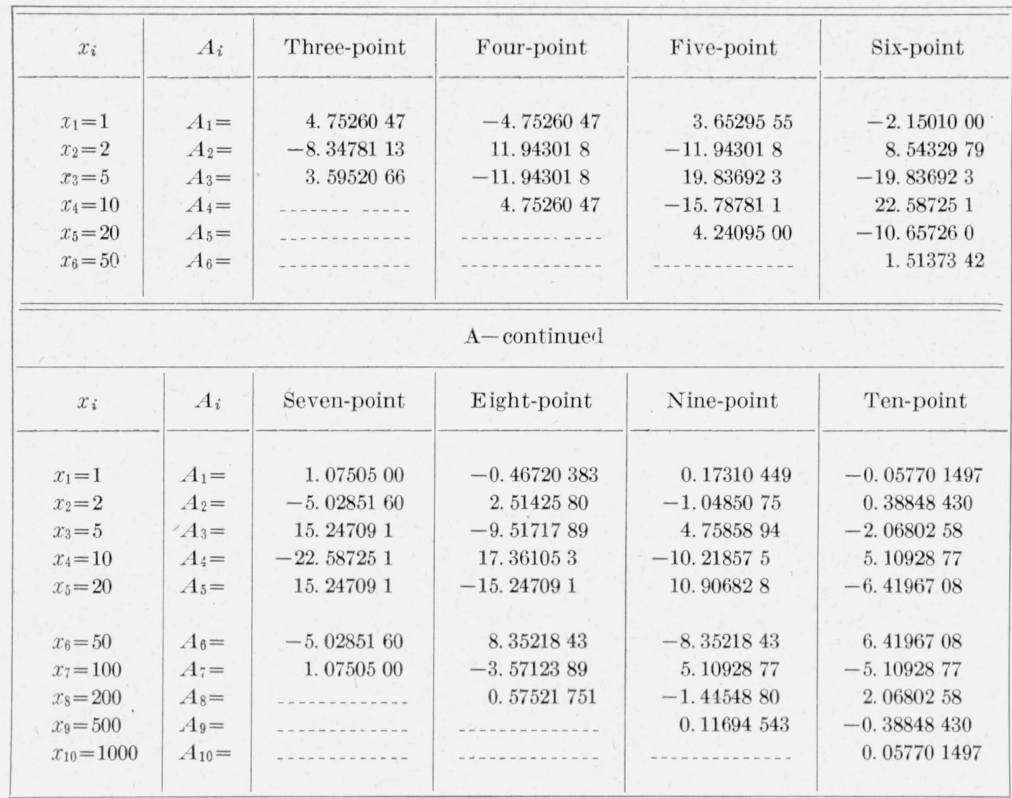

B

\begin{tabular}{|c|c|c|c|c|c|c|}
\hline$x_{i}$ & $A_{i}$ & Three-point & Four-point & Five-point & Six-point & Seven-point \\
\hline$x_{1}=1$ & $A_{1}=$ & 1. 4306766 & -0.84208465 & 0.42104232 & -0.15600111 & 0. 052000371 \\
\hline$x_{2}=5$ & $A_{2}=$ & $-4.75260 \quad 47$ & 4. 7526047 & -3.6529555 & 1. 8264777 & -0.79376529 \\
\hline$x_{3}=10$ & $A_{3}=$ & 3.3219281 & -4.7526047 & 4. 7526047 & -2.7973447 & 1. $39867 \quad 23$ \\
\hline$x_{4}=50$ & $A_{4}=$ & - n.t. & 0.84208465 & $-2.79734 \quad 47$ & 2. 7973447 & -2.1501000 \\
\hline$x_{5}=100$ & $A_{5}=$ & & ... & 1. 2766532 & -1.8264777 & 1. 8264777 \\
\hline$x_{6}=500$ & $A_{6}=$ & & -..... & - & 0.15600111 & -0.51822448 \\
\hline$x_{7}=1000$ & $A_{7}=$ & - & - n. & - n & - & 0.18493938 \\
\hline
\end{tabular}

C

\begin{tabular}{|c|c|c|c|c|c|c|}
\hline$x_{i}$ & $A_{i}$ & Three-point & Four-point & Five-point & Six-point & Seven-point \\
\hline$x_{1}=1$ & $A_{1}=$ & 3. 3219281 & -2.5533063 & 1. 2766532 & -0.55481 ४13 & 0.18493938 \\
\hline$x_{2}=2$ & $A_{2}=$ & -4.7526047 & 4. 7526047 & $-2.79734 \quad 47$ & 1. 3986723 & -0.51822448 \\
\hline$x_{3}=10$ & $A_{3}=$ & 1. 4306766 & -4.7526047 & 4. 7526047 & -3.6529555 & 1.8264777 \\
\hline$x_{4}=20$ & $A_{4}=$ & - . & 2. 5533063 & -3.6529555 & 3.6529555 & -2.1501000 \\
\hline$x_{5}=100$ & $A_{5}=$ & & $\ldots$ & c. 42104232 & -1.3986723 & 1. 3986723 \\
\hline$x_{6}=200$ & $A_{6}=$ & - & - & - & 0.55481813 & -0.79376529 \\
\hline$x_{7}=1000$ & $A_{7}=$ & - & - & - & - & 0. 052000371 \\
\hline
\end{tabular}


by the $A_{i}$ 's for a certain $n$, we use a different set of $A_{i}$ 's corresponding to $n-1$, for interpolation.

\section{Interpolation}

Once we have ascertained, by use of table 1 , that a function is adequately represented by a polynomial of degree $n-1$ in $\log x$ (remembering that $n-1$ is here the $n-2$ of III.), we can interpolate by use of a rearrangement of Lagrange's formula, which was suggested by a paper of W. J. Taylor [4]. The original method and notation of Taylor are not described in this article because he developed them only for the special case of equally spaced arguments, and here they would be superfluous. The interpolation formula is

$$
f(x) \sim\left(\sum_{i=1}^{n} a_{i} f\left(x_{i}\right)\right) / \sum_{i=1}^{n} a_{i},
$$

where

$$
a_{i}=A_{i} /\left(\log x-\log x_{i}\right)
$$

and $A_{i}$ is given by (1).

\section{Logarithms to Other Bases}

The coefficients $A_{i}$ have been computed from common (base 10) logarithms, according to (1). It would have been possible, instead, to have the $A_{i}$ 's calculated for any other logarithmic base. Furthermore, it is permissible when computing the $a_{i}$ 's from (3), to use logarithms to a base different from that underlying the $A_{i}$ 's. To illustrate this point, natural logarithms (to the base $e=2.71828$ 183) have been used in the two examples in section VIII. Extensive tables of $\log _{\mathrm{e}} x$ are given in [5].

\section{Conversion to Arguments in Geometric Progression}

If a large number of interpolations are required for a function given for any one set of values $x_{i}$, it may be convenient to prepare, by the method in section IV, an auxiliary table giving the function at a new set of values $y_{i}$, where the $y_{i}$ are now in geometric progression. Then we can use ordinary interpolation formulae and coefficients, as suggested in section II. This point was called to the writer's attention by Churchill Eisenhart and Julius Lieblein, of the Statistical Engineering Laboratory.

\section{Use of $A_{i}$ for Other Arguments}

If the given set of values $x_{i}$ does not begin with 1 , the table of coefficients $A_{i}$ may sometimes still be used by a suitable change of the independent variable $x$. Notice that a polynomial in log $(a x)$ or $\log$ $(b / x)$ is still a polynomial in $\log x$. Thus, for instance, if a function is given at the points $x=10,20,50$ and 100 , we may consider it as a function of $x^{\prime}=100 / x$, given at the points $x^{\prime}=1,2,5$ and 10 , so that table $1, \mathrm{~A}$, may be used. Again, if a function is given at the points $x=20,100,200$ and 1000 , it may, instead, be considered as a function of $x^{\prime}=1000 / x$ given at the points $x^{\prime}=1,5,10$ and 50 , so that table $1, B$, may be used. Schedules A, B, and C list such transformations of the independent variable, giving both the sets of $x_{i}$ for which the change of variable to $1 / x$ is applicable, and the new arguments $x_{i}^{\prime}$, which are proportional to $1 / x_{i}$.

Transformation schedules

\begin{tabular}{|c|c|c|c|c|c|c|c|c|c|}
\hline$x_{i}$ & $x_{i}{ }^{\prime}$ & $x_{i}$ & $x_{i}{ }^{\prime}$ & $x_{i}$ & $x_{i}^{\prime}$ & $x_{i}$ & $x_{i}^{\prime}$ & $x_{i}$ & $x_{i}{ }^{\prime}$ \\
\hline $\begin{array}{r}2 \\
5 \\
10\end{array}$ & $\begin{array}{l}5 \\
2 \\
1\end{array}$ & $\begin{array}{r}2 \\
5 \\
10 \\
20 \\
50 \\
100\end{array}$ & $\begin{array}{r}50 \\
20 \\
10 \\
5 \\
2 \\
1\end{array}$ & $\begin{array}{r}2 \\
5 \\
10 \\
20 \\
50 \\
100 \\
200 \\
500 \\
1000\end{array}$ & $\begin{array}{r}500 \\
200 \\
100 \\
50 \\
20 \\
10 \\
5 \\
2 \\
1\end{array}$ & $\begin{array}{r}5 \\
10 \\
20 \\
50 \\
100\end{array}$ & $\begin{array}{r}20 \\
10 \\
5 \\
2 \\
1\end{array}$ & $\begin{array}{r}5 \\
10 \\
20 \\
50 \\
100 \\
200 \\
500 \\
1000\end{array}$ & $\begin{array}{r}200 \\
100 \\
50 \\
20 \\
10 \\
5 \\
2 \\
1\end{array}$ \\
\hline
\end{tabular}

Schedule A.

\begin{tabular}{|c|c|c|c|c|c|c|c|}
\hline$x_{i}$ & $x_{i}^{\prime}$ & $x_{i}$ & $x_{i}^{\prime}$ & $x_{i}$ & $x_{i}{ }^{\prime}$ & $x_{i}$ & $x_{i}{ }^{\prime}$ \\
\hline $\begin{array}{r}2 \\
10 \\
20 \\
100\end{array}$ & $\begin{array}{r}50 \\
10 \\
5 \\
1\end{array}$ & $\begin{array}{r}2 \\
10 \\
20 \\
100 \\
200 \\
1000\end{array}$ & $\begin{array}{r}500 \\
100 \\
50 \\
10 \\
5 \\
1\end{array}$ & $\begin{array}{r}5 \\
10 \\
50\end{array}$ & $\begin{array}{r}10 \\
5 \\
1\end{array}$ & $\begin{array}{r}5 \\
10 \\
50 \\
100 \\
500\end{array}$ & $\begin{array}{r}100 \\
50 \\
10 \\
5 \\
1\end{array}$ \\
\hline
\end{tabular}

\begin{tabular}{|c|c|c|c|c|c|c|c|}
\hline$x_{i}$ & $x_{i}{ }^{\prime}$ & $x_{i}$ & $x_{i}{ }^{\prime}$ & $x_{i}$ & $x_{i}^{\prime}$ & $x_{i}$ & $x_{i}{ }^{\prime}$ \\
\hline $\begin{array}{r}5 \\
10 \\
50 \\
100\end{array}$ & $\begin{array}{r}20 \\
10 \\
2 \\
1\end{array}$ & $\begin{array}{r}5 \\
10 \\
50 \\
100 \\
500 \\
1000\end{array}$ & $\begin{array}{r}200 \\
100 \\
20 \\
10 \\
2 \\
1\end{array}$ & $\begin{array}{r}2 \\
10 \\
20\end{array}$ & $\begin{array}{r}10 \\
2 \\
1\end{array}$ & $\begin{array}{r}2 \\
10 \\
20 \\
100 \\
200\end{array}$ & $\begin{array}{r}100 \\
20 \\
10 \\
2 \\
1\end{array}$ \\
\hline
\end{tabular}

\section{Illustrations of Use of Tables}

The following two illustrative examples show how to use the tables of $A_{i}$.

Example 1. Given

\begin{tabular}{|r|r||r|r|}
\hline \multicolumn{1}{r|}{$x_{i}$} & \multicolumn{1}{|c|}{$f\left(x_{i}\right)$} & \multicolumn{1}{|c|}{$x_{i}$} & \multicolumn{1}{|c|}{$f\left(x_{i}\right)$} \\
\cline { 2 - 2 } & & & \\
\cline { 2 - 3 } 1 & & & \\
2 & 1.52 & 50 & 6333.56 \\
5 & 50.30 & 100 & 15752.58 \\
10 & 343.74 & 200 & 34648.66 \\
20 & 1447.15 & 1000 & 85417.78 \\
& & & 155833.72 \\
\hline
\end{tabular}


To calculate $f(18)$. In the absence of knowledge of an explicit formula for $f(x)$, we find that its seventh divided difference, employing the coefficients $A_{i}$ in table 1, A, for the eight points from 1 to 200 , is less than 0.05 in absolute value. This indicates that the function behaves, up to the last place, like a sixth degree polynomial in $\log x$ (any base). Hence only seven points are needed for the interpolation. The work may be arranged in the following way: (Note that an extra place is carried in some columns to reduce the error that would arise in rounding before the final answer is reached.)

\begin{tabular}{|c|c|c|c|}
\hline$x_{i}$ & $\log _{e} 18-\log _{e} x_{i}$ & $a_{i} \equiv A_{i} /\left(\log _{e} 18-\log _{e} x_{i}\right)$ & $a_{i} f\left(x_{i}\right)$ \\
\hline \multirow[t]{2}{*}{$\begin{array}{r}1 \\
2 \\
5 \\
10 \\
20 \\
50 \\
100\end{array}$} & 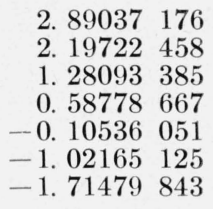 & $\begin{array}{r}0.371942 \\
-2.288576 \\
11.903106 \\
-38.427634 \\
-144.713527 \\
4.921950 \\
-0.626925\end{array}$ & $\begin{array}{r}0.193 \\
-2.540 \\
598.726 \\
-13209.115 \\
-209422.181 \\
31173.466 \\
-9875.686\end{array}$ \\
\hline & . & $\sum_{i=1}^{7} a_{i}=-168.859664$ & $\begin{array}{l}\sum_{i=1}^{7} a_{i} f\left(x_{i}\right)=-200737.137 \\
\sum a_{i} f\left(x_{i}\right) / \sum a_{i}=1188.78\end{array}$ \\
\hline
\end{tabular}

The answer found here, 1188.78 , is correct to its last significant figure. The function $f(x)$ was chosen to be $\left(\log _{e} x\right)^{6}+3\left(\log _{e} x\right)^{5}+\frac{\pi}{6}$.

Example 2. Given

\begin{tabular}{|r|r|r|}
\hline$x_{i}$ & $x_{i}{ }^{\prime}$ & $f\left(x_{i}\right)$ or $\bar{f}\left(x_{i}{ }^{\prime}\right)$ \\
\cline { 2 - 3 } & & \\
\hline 20 & 50 & 15.45981 \\
100 & 10 & 40.07131 \\
200 & 5 & 54.18217 \\
1000 & 1 & 95.09949 \\
\hline
\end{tabular}

To calculate $f(160)$. To use the present tables of coefficients $\mathrm{A}_{i}$ it is necessary to change the variable to $x^{\prime}=1000 / x$. (See Transformation schedule B.) If $f(x)$ is called $\bar{f}\left(x^{\prime}\right)$, then $f(160)=\bar{f}(6.25)$. As in the previous example, in the absence of knowledge of an explicit formula for $f(x)$, we find that the absolute value of its third divided difference, employing the coefficients $A_{i}$ in table $1, \mathrm{~B}$, for four points, is less than $1 \frac{1}{2}$ units in the fifth decimal place. This indicates that the function behaves, up to the last place, like a second degree polynomial in $\log x$. Hence only three points are needed for the interpolation, which is carried out as before:

\begin{tabular}{|c|c|c|c|}
\hline$x_{i}{ }^{\prime}$ & $\log _{e} 6.25-\log _{e} x_{i}{ }^{\prime}$ & $a_{i} \equiv A_{i} /\left(\log _{e} 6.25-\log _{e} x_{i}{ }^{\prime}\right)$ & $a_{i} \bar{f}\left(x_{i}{ }^{\prime}\right)$ \\
\hline \multirow[t]{2}{*}{$\begin{array}{r}1 \\
5 \\
10\end{array}$} & \multirow[t]{2}{*}{$\begin{array}{r}\text { 1. } 83258146 \\
0.22314355 \\
-0.47000363\end{array}$} & $\begin{array}{r}0.7806892 \\
-21.2984184 \\
-7.06787 \quad 75\end{array}$ & $\begin{array}{r}74.24314 \\
-1153.99453 \\
-283.21911\end{array}$ \\
\hline & & $\sum_{i=1}^{3} a_{i}=-27.5856067$ & $\begin{array}{c}\sum_{i=1}^{3} a_{i} \bar{f}\left(x_{i}{ }^{\prime}\right)=-1362.97050 \\
\sum a_{i} \bar{f}\left(x_{i}{ }^{\prime}\right) / \sum a_{i}=49.40876\end{array}$ \\
\hline
\end{tabular}

This answer, 49.40876, is also correct to the last figure given, the function $f(x)$ having been chosen to be $2.20\left(\log _{e} x\right)^{2}-1.43 \log _{e} x$.

The author expresses his appreciation to Churchill Eisenhart and Julius Lieblein for their thorough study and constructive criticism of this paper in its original form, and to John Todd and Franz L. Alt for their editorial review and for suggesting some final improvements in the presentation.

\section{References}

[1] Tables of Lagrangian interpolation coefficients. (Columbia University Press, New York, 1944.)

[2] L. M. Milne-Thomson, Calculus of finite differences, ch. I, p. 1 (Macmillan and Co., London, 1933).

[3] E. T. Whittaker and G. Robinson, Calculus of observations, ch. II, p. 20 (Blackie and Son, London, 1944).

[4] W. J. Taylor, A method of Lagrangian curvilinear interpolation, J.- Research NBS 35, 151 (1945) RP1667.

[5] Table of natural logarithms, vol. I to IV, Mathematical Tables NBS, MT7, MT9, MT10, MT12.

Washington, February 13, 1950. 